\title{
(6) OPEN ACCESS \\ Anatomical effects of dexamethasone intravitreal implant in diabetic macular oedema: a pooled analysis of 3-year phase III trials
}

\author{
Ronald P Danis, ${ }^{1}$ Srinivas Sadda, ${ }^{2}$ Xiao-Yan Li, ${ }^{3}$ Harry Cui, ${ }^{3}$ Yehia Hashad, ${ }^{3}$ \\ Scott M Whitcup ${ }^{3}$
}

- Additional material is published online only. To view please visit the journal online (http://dx.doi.org/10.1136/ bjophthalmol-2015-306823).

${ }^{1}$ Department of Ophthalmology and Visual Sciences, University of Wisconsin-Madison Madison, Wisconsin, USA ${ }^{2}$ Doheny Image Reading Center, Doheny Eye Institute, Los Angeles, California, USA ${ }^{3}$ Allergan, Inc., Irvine, California, USA

\section{Correspondence to} Dr Ronald P Danis, Department of Ophthalmology and Visual Sciences, Fundus Photograph Reading Center, University of Wisconsin-Madison, 2870 University Ave, Suite 102, Madison, WI 53705, USA: rpdanis@wisc.edu

Received 27 February 2015 Revised 27 August 2015 Accepted 3 September 2015 Published Online First 18 November 2015

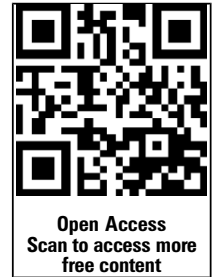

CrossMark

To cite: Danis RP, Sadda $S$,

Li X-Y, et al. Br J

Ophthalmol 2016;100:

796-801.

\section{ABSTRACT}

Background/aim To assess long-term effects of dexamethasone intravitreal implant (DEX implant) monotherapy on retinal morphology in diabetic macular oedema (DME).

Methods Two multicentre, masked, phase III studies with identical protocols randomised patients with $\mathrm{DME}_{\text {, }}$ best-corrected visual acuity of 34-68 Early Treatment Diabetic Retinopathy Study letters and central subfield retinal thickness (CSRT) $\geq 300 \mu \mathrm{m}$ to DEX implant 0.7, $0.35 \mathrm{mg}$ or sham procedure. Patients were followed up for 3 years (39 months if treated at month 36), with retreatment allowed at $\geq 6$-month intervals. Patients needing other macular oedema (ME) therapy exited the study. Changes from baseline in CSRT, macular volume and ME grade, area of retinal thickening, macular leakage, macular capillary loss and diabetic retinopathy severity were assessed.

Results After 3 years, more eyes treated with DEX implant 0.7 and $0.35 \mathrm{mg}$ than sham showed improvement (although small) in ME grade ( $p<0.05$ vs sham). DEX implant $0.7 \mathrm{mg}$ delayed time to onset of two-step progression in diabetic retinopathy severity by $\sim 12$ months. DEX implant 0.7 and $0.35 \mathrm{mg}$ produced small, non-sustained reductions in macular leakage but had no significant effect on macular capillary loss.

Conclusions DEX implant 0.7 or $0.35 \mathrm{mg}$, administered at $\geq 6$-month intervals over 3 years, produced sustained retinal structural improvement in DME.

Trial registration number NCT00168337 and NCT00168389.

\section{INTRODUCTION}

Over the last decade, new therapeutic approaches have resulted from improved understanding of the pathophysiological processes responsible for endothelial blood-retinal barrier breakdown in diabetic macular oedema (DME). ${ }^{1}$ Several inter-related ocular inflammatory events are of particular relevance: the release of vascular permeability factors such as vascular endothelial growth factor (VEGF); upregulation of inflammatory mediators; increased expression of endothelial adhesion molecules and the influx and adhesion of leucocytes to the retinal microvasculature (leukostasis), resulting in endothelial cell injury and apoptosis. ${ }^{2-5}$ Several VEGF inhibitors, including aflibercept, bevacizumab, pegaptanib and ranibizumab, have shown clinical efficacy as intravitreal therapies for DME. ${ }^{6} 7$ Current treatment guidelines recognise the role of ranibizumab in improving visual acuity in patients with macular centre involvement and vision loss due to DME. ${ }^{8}$ However, per-protocol clinical use of VEGF inhibitors in DME requires monthly intravitreal injections. ${ }^{8}$ Moreover, many patients exhibit retinal thickening despite anti-VEGF therapy, highlighting the need for additional treatments.

Intravitreal corticosteroids downregulate expression of cytokines such as tumour necrosis factor- $\alpha,{ }^{9}$ nuclear factor- $\mathrm{kB},{ }^{9} \mathrm{VEGF}^{9}$ and intercellular adhesion molecule-1, ${ }^{9} 10$ and inhibit leukostasis and retinal microvasculature leakage. ${ }^{9}{ }^{10}$ Fluocinolone acetonide (Retisert, Bausch \& Lomb, Bridgewater, New Jersey, USA; Iluvien, Alimera Sciences, Alpharetta, Georgia, USA) and dexamethasone (DEX implant; Ozurdex, Allergan, Irvine, California, USA) are available commercially as slow-release intravitreal implant systems. DEX implant has recently been approved for the treatment of adults with DME. In early-phase clinical trials in DME, DEX implant monotherapy ${ }^{11}$ and combination laser therapy ${ }^{12}$ were effective in improving central subfield retinal thickness (CSRT) and best-corrected visual acuity (BCVA). These findings have recently been confirmed in two pivotal phase III trials of DEX implant in DME (the MEAD studies). In a pooled analysis of the MEAD study data, DEX implant met the primary efficacy end point of $\geq 15$-letter improvement in BCVA and showed acceptable tolerability. ${ }^{13}$ The anatomical findings from the MEAD studies are detailed here.

\section{METHODS}

Study design and participants

Data collected from two identically designed, 3-year, multicentre, masked, phase III trials (NCT00168337 and NCT00168389) of the safety and efficacy of DEX implant in the treatment of DME (MEAD trials) were pooled. The trials were conducted at 131 sites in 22 countries worldwide between February 2005 and June 2012. The protocol was approved by the institutional review board/ ethics committee at each study site, and the trials were conducted in accordance with the Declaration of Helsinki. All patients provided written informed consent. The protocol is described in detail elsewhere ${ }^{13}$ and is summarised below.

Adults with diabetic retinopathy-associated macular oedema involving the fovea, previously treated with medical or laser therapy, and an Early Treatment Diabetic Retinopathy Study (ETDRS) BCVA in the study eye of 34-68 letters (20/200 20/50) were enrolled. Treatment-naïve patients 
refusing laser treatment or who, in the investigator's opinion, would not benefit from laser treatment also were eligible. Retinal thickness in the central $1 \mathrm{~mm}$ macular subfield measured by time-domain optical coherence tomography (TD-OCT; Stratus OCT3 or OCT2 (used for only 1\% of study submissions), Carl Zeiss Meditec, Dublin, California, USA) was required to be $\geq 300 \mu \mathrm{m}$ in the study eye. Key exclusion criteria were uncontrolled diabetes (glycosylated haemoglobin $>10 \%$ ); glaucoma; ocular hypertension (untreated intraocular pressure $>23 \mathrm{~mm} \mathrm{Hg}$ ); aphakia; active iris or retinal neovascularisation; history of pars plana vitrectomy or steroid-induced ocular hypertension; recent intraocular laser or incisional surgery or intravitreal VEGF inhibitor or triamcinolone treatment; and current use of systemic steroids.

Patients were randomised $(1: 1: 1)$ to intravitreal DEX implant $0.7 \mathrm{mg}$, DEX implant $0.35 \mathrm{mg}$ or a sham procedure in the study eye. If both eyes were eligible, the eye with the shorter duration of macular oedema was selected. Patients were evaluated for retreatment every 3 months during the 3 -year study, but retreatment could not be performed more often than every 6 months. Retreatment was allowed if retinal thickness in the $1 \mathrm{~mm}$ central macular subfield was $>225 \mu \mathrm{m}$ (revised to $>175 \mu \mathrm{m}$ or evidence of residual oedema in a protocol amendment in 2010). Patients needing adjunctive or other therapy for macular oedema were required to exit the study prior to administration of additional treatment. Efficacy data captured before patient exit were included in the analysis.

\section{Study assessments and end points}

The primary efficacy end point in the MEAD trials was the percentage of patients with $\geq 15$-letter improvement in BCVA from baseline in the study eye at final assessment (end of year 3 or 39 months for patients treated at month 36). Prespecified secondary end points included changes in retinal anatomy measured using OCT, fundus photography and fluorescein angiography. Image evaluation (grading) was performed at a centralised reading facility (University of Wisconsin Fundus Photograph Reading Center, Madison, Wisconsin, USA) by certified masked technicians.

TD-OCT (Stratus OCT3 or, if unavailable, OCT2) was conducted at 3-month intervals. Six radial scans, each $\sim 6 \mathrm{~mm}$ long and centred on the fovea and performed using fast macular thickness map settings (128 A-scans/B-scan), were supplemented by high-resolution $6 \mathrm{~mm}$ cross-hair scans (512 A-scans/B-scan). Stereoscopic $30^{\circ}$ or $35^{\circ}$ colour fundus photographs of the study eye were taken at baseline, every 3 months during the first year and every 6 months during the second and third years. Fundus photographs were assessed for presence and extent of retinal thickening, diabetic retinopathy severity level and presence of clinically significant macular oedema (CSME). Diabetic retinopathy was graded using the ETDRS Final Retinopathy Severity Scale condensed to nine severity categories. ${ }^{14}$ Outcomes of interest included changes from baseline in disc area of central retinal thickening and macular oedema grade (improvement, no change or worsening).

Fluorescein angiography was performed at baseline, months 6, 12 and 24 and at the end of year 3 (or 39 months for patients treated at month 36) to assess macular fluorescein leakage and perifoveal capillary integrity. Grading protocols were adapted from the ETDRS clinical trials. ${ }^{15}$ The mean change from baseline to study end in total disc area of macular capillary loss and the proportions of patients with and without ischaemia (defined as a total area of macular capillary loss $>0.5$ disc area) at baseline and the last visit were determined. Details of the assessments are described in the online supplementary materials.

\section{Statistical analyses}

Unless stated otherwise, all efficacy analyses were performed with missing values imputed by last observation carried forward (LOCF) for the intent-to-treat (ITT) population (all randomised patients). Treatment comparisons and estimates based on LOCF were supported by sensitivity analyses using multiple imputation. Area under the curve (AUC) analysis of the average change from baseline in CSRT during the study used observed values in the ITT population; missing values were not imputed. Areas of central retinal thickening, fluorescein leakage and macular capillary loss and changes from baseline in CSRT and macular volume were analysed using analysis of covariance with baseline value as a covariate. Changes from baseline in proportions of patients with CSME and central retinal thickening were compared using Wilcoxon rank-sum test. Proportions of patients in each diabetic retinopathy severity category and proportions of patients with $\geq 2$-step progression from baseline in diabetic retinopathy severity category were analysed using the CochranMantel-Haenszel general association test stratified by study. Time to two-step progression in diabetic retinopathy severity category was analysed using the Kaplan-Meier method, and cumulative progression rates were compared using the log-rank test. All statistical tests were two-sided and performed at $\alpha=0.05$ significance level. SAS V.9.3 (SAS Institute, Cary, North Carolina, USA) was used.

\section{RESULTS}

\section{Study population}

The pooled ITT population comprised 1048 randomised patients (table 1), of whom 607 patients (57.9\%) completed all visits. Completion rates were appreciably higher in the DEX implant $0.7 \mathrm{mg}(64.1 \%)$ and $0.35 \mathrm{mg}(66.3 \%)$ groups than in the sham group (43.4\%).

The median number of study treatments administered in each DEX implant group ranged between 4 and 5 compared with 3 in the sham group. Baseline demographics and study eye characteristics did not differ significantly among the three treatment groups (table 1).

\section{OCT findings}

DEX implant-treated eyes showed marked fluctuation in the reduction in CSRT at consecutive study visits, particularly during year 1 , creating a saw-tooth pattern of treatment effect (figure $1 ;<1 \%$ of scans were deemed non-gradable). Similar results were obtained with the observed data analysis. Study eyes treated with DEX implant showed greater reductions from baseline in CSRT than sham-treated eyes at all time points (figure 1).

At the final study visit, CSRT was reduced by (mean) 117.3 and $127.8 \mu \mathrm{m}$ in DEX implant $0.7-$ and $0.35 \mathrm{mg}$-treated eyes versus $62.1 \mu \mathrm{m}$ in sham-treated eyes (both $\mathrm{p}<0.001$ vs sham; table 2). Over the full study period, the mean average reduction in CSRT (AUC approach) was greater in DEX implant-treated eyes (table 2). At all study time points from month 3 onward, the proportion of study eyes with CSRT $>250 \mu \mathrm{m}$ was lower in the DEX implant than the sham treatment groups. At the final study visit, the decline in the proportion of study eyes in this category was greater with DEX implant $0.7 \mathrm{mg}$ (from $94.5 \%$ (baseline) to $60.2 \%$ ) and DEX implant $0.35 \mathrm{mg}$ (from $94.8 \%$ to $58.7 \%$ ) than with sham (from $95.9 \%$ to $71.6 \%$ ). Likewise, at all study time points DEX implant-treated eyes displayed greater 
Table 1 Baseline demographic and study eye characteristics of the ITT population

\begin{tabular}{|c|c|c|c|}
\hline Characteristic & DEX implant $0.7 \mathrm{mg}$ & DEX implant $0.35 \mathrm{mg}$ & Sham procedure \\
\hline \multicolumn{4}{|l|}{ Demographic characteristics } \\
\hline Age, years & $N=351$ & $N=347$ & $N=350$ \\
\hline Mean (SD) & $62.5(8.3)$ & $62.3(9.2)$ & $62.5(9.5)$ \\
\hline Range & $33-85$ & $25-84$ & $26-88$ \\
\hline Gender, n (\%) & $\mathrm{N}=351$ & $\mathrm{~N}=347$ & $\mathrm{~N}=350$ \\
\hline Male & $213(60.7)$ & $206(59.4)$ & $217(62.0)$ \\
\hline Race/ethnicity, n (\%) & $\mathrm{N}=351$ & $\mathrm{~N}=347$ & $\mathrm{~N}=350$ \\
\hline Asian & $55(15.7)$ & $58(16.7)$ & $54(15.4)$ \\
\hline Black & $16(4.6)$ & $16(4.6)$ & $20(5.7)$ \\
\hline Caucasian & $234(66.7)$ & $234(67.4)$ & $233(66.6)$ \\
\hline Hispanic & $35(10.0)$ & $34(9.8)$ & $33(9.4)$ \\
\hline Other & $11(3.1)$ & $5(1.4)$ & $10(2.9)$ \\
\hline Diabetes type, n (\%) & $N=351$ & $\mathrm{~N}=347$ & $\mathrm{~N}=350$ \\
\hline Type 1 & $34(9.7)$ & $22(6.3)$ & $28(8.0)$ \\
\hline Type 2 & $314(89.5)$ & $325(93.7)$ & $322(92.0)$ \\
\hline Not available & $3(0.9)$ & 0 & 0 \\
\hline Duration of diabetes, years & $N=349$ & $\mathrm{~N}=347$ & $N=348$ \\
\hline Mean (SD) & $16.5(9.0)$ & $15.8(9.4)$ & $15.9(9.1)$ \\
\hline $\mathrm{HbA1c}, \%$ & $\mathrm{~N}=347$ & $\mathrm{~N}=345$ & $\mathrm{~N}=349$ \\
\hline Mean (SD) & $7.6(1.2)$ & $7.5(1.1)$ & $7.5(1.1)$ \\
\hline \multicolumn{4}{|l|}{ Study eye characteristics } \\
\hline ETDRS letter score & $N=351$ & $\mathrm{~N}=347$ & $N=350$ \\
\hline Mean (SD) & $56.1(9.9)$ & $55.5(9.7)$ & $56.9(8.7)$ \\
\hline CSRT, $\mu \mathrm{m}$ & $\mathrm{N}=348$ & $\mathrm{~N}=344$ & $\mathrm{~N}=342$ \\
\hline Mean (SD) & $463.0(157.1)$ & $466.8(159.5)$ & $460.9(132.6)$ \\
\hline Study eyes with CSRT $>250 \mu \mathrm{m}, \%$ & 94.5 & 94.8 & 95.9 \\
\hline Duration of DME, months & $\mathrm{N}=350$ & $\mathrm{~N}=347$ & $\mathrm{~N}=349$ \\
\hline Mean (SD) & $23.6(26.0)$ & $25.2(31.4)$ & $25.9(27.3)$ \\
\hline Range & $0-163$ & 0-299 & $0-187$ \\
\hline DME classification, $\mathrm{n}(\%)^{*}$ & $\mathrm{~N}=351$ & $\mathrm{~N}=347$ & $\mathrm{~N}=350$ \\
\hline Focal & $127(36.2)$ & $136(39.2)$ & $142(40.6)$ \\
\hline Intermediate & $134(38.2)$ & $124(35.7)$ & $122(34.9)$ \\
\hline Diffuse & $69(19.7)$ & $60(17.3)$ & $72(20.6)$ \\
\hline Not available & $16(4.6)$ & $25(7.2)$ & $10(2.9)$ \\
\hline None & $5(1.4)$ & $2(0.6)$ & $4(1.1)$ \\
\hline Previous DME treatment, $\mathrm{n}(\%)$ & $\mathrm{N}=351$ & $\mathrm{~N}=347$ & $\mathrm{~N}=350$ \\
\hline Focal/grid laser & $231(65.8)$ & $224(64.6)$ & $243(69.4)$ \\
\hline Intravitreal steroid & $58(16.5)$ & $69(19.9)$ & $61(17.4)$ \\
\hline Anti-VEGF & $25(7.1)$ & 39 (11.2) & $26(7.4)$ \\
\hline None & $104(29.6)$ & $98(28.2)$ & $89(25.4)$ \\
\hline Severity of NPDR, $n(\%)$ & $\mathrm{N}=351$ & $\mathrm{~N}=347$ & $\mathrm{~N}=350$ \\
\hline Moderate or better & $173(49.3)$ & $170(49.0)$ & $174(49.7)$ \\
\hline Severe or worse & $151(43.0)$ & $151(43.5)$ & $149(42.6)$ \\
\hline Not available & $27(7.7)$ & $26(7.5)$ & $27(7.7)$ \\
\hline Macular perfusion status, n (\%) & $\mathrm{N}=351$ & $\mathrm{~N}=347$ & $\mathrm{~N}=350$ \\
\hline Ischaemict & $43(12.3)$ & $31(8.9)$ & $27(7.7)$ \\
\hline Non-ischaemic & $257(73.2)$ & $260(74.9)$ & $284(81.1)$ \\
\hline Not available & $51(14.5)$ & $56(16.1)$ & $39(11.1)$ \\
\hline Lens status, n (\%) & $N=351$ & $\mathrm{~N}=347$ & $\mathrm{~N}=350$ \\
\hline Phakic & $265(75.5)$ & $259(74.9)$ & $249(71.1)$ \\
\hline Pseudophakic & $86(24.5)$ & $88(25.4)$ & $101(28.9)$ \\
\hline
\end{tabular}

*DME classification based upon clinical assessment by the treating physician.

†Area of macular capillary loss on fluorescein angiography $>0.5$ disc areas. Analysis based on Fisher exact test.

Intergroup comparisons performed using Pearson's $\chi^{2}$ test (categorical variables) and a one-way analysis of variance model (continuous variables).

CSRT, central subfield retinal thickness; DEX implant, dexamethasone intravitreal implant; DME, diabetic macular oedema; ETDRS, Early Treatment Diabetic Retinopathy Study; HbA1c,

glycosylated haemoglobin; ITT, intent-to-treat; NPDR, non-proliferative diabetic retinopathy; VEGF, vascular endothelial growth factor.

reductions from baseline in macular volume than sham-treated eyes (both doses $\mathrm{p}<0.001$ vs sham at study end; figure 2 and table 2).

\section{Fundus photography findings}

The area of central retinal thickening showed greater reduction from baseline in DEX implant-treated versus sham-treated eyes 
Figure 1 Mean change from baseline in retinal thickness in the central subfield versus time. $\mathrm{p} \leq 0.024$ at all time points for dexamethasone intravitreal (DEX) implants versus sham (analysis of covariance with treatment value as covariate). $B$, baseline; $F$, final visit. and study as fixed effects and baseline

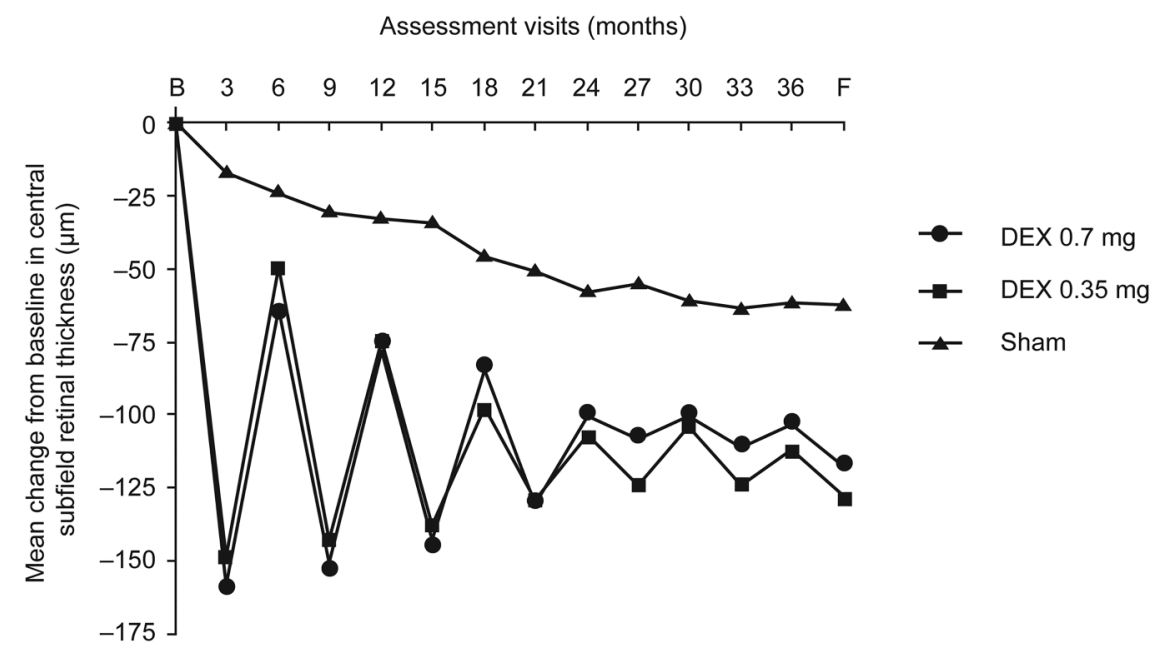

at all study time points; at study end, mean reductions of 2.75 and 2.93 disc areas were recorded in DEX implant 0.7- and $0.35 \mathrm{mg}$-treated eyes, respectively (both $\mathrm{p}<0.001$ vs sham; table 2). The corresponding absolute area of central retinal thickening at study end was (mean) $5.34,5.38$ and 6.13 disc areas, respectively.

Table 2 Summary of optical coherence tomography, fundus photography and fluorescein angiography findings

\begin{tabular}{|c|c|c|c|}
\hline End point & DEX implant $0.7 \mathrm{mg}$ & DEX implant $0.35 \mathrm{mg}$ & Sham procedure \\
\hline \multicolumn{4}{|l|}{ Optical coherence tomography } \\
\hline Retinal thickness in central subfield, $\mu \mathrm{m}$ & $\mathrm{N}=348$ & $\mathrm{~N}=344$ & $\mathrm{~N}=342$ \\
\hline $\mathrm{BL}$, mean (SD) & $463.0(157.1)$ & $466.8(159.5)$ & $460.9(132.6)$ \\
\hline Change from BL to month 39 , mean (SD) & $-117.3(208.1)^{* * *} \dagger$ & $-127.8(196.7)^{* * *} \dagger$ & $-62.1(180.1)$ \\
\hline AUC change from BL during study, mean (SD) & $-111.6(134.1)^{* * *} \dagger$ & $-107.9(135.8)^{* * *} \dagger$ & $-41.9(116.0)$ \\
\hline Macular volume, $\mathrm{mm}^{3}$ & $\mathrm{~N}=264$ & $\mathrm{~N}=245$ & $\mathrm{~N}=250$ \\
\hline $\mathrm{BL}$, mean (SD) & $9.73(2.07)$ & $9.81(2.15)$ & $9.44(1.85)$ \\
\hline Change from BL to month 39 , mean (SD) & $-1.06(2.22)^{* * *} \ddagger$ & $-1.14(1.75)^{* * *} \ddagger$ & $-0.31(1.62)$ \\
\hline \multicolumn{4}{|l|}{ Fundus photography } \\
\hline Patients with CSME, \%§ & $N=313-340$ & $N=308-336$ & $N=307-340$ \\
\hline Baseline & 96.8 & $98.7^{*} \emptyset$ & 95.4 \\
\hline Month 39 & $77.1 * q$ & $75.9 * \star \emptyset$ & 84.2 \\
\hline Patients with improvement from BL at month 39 & $20.4^{*} ף$ & $22.4^{* * \pi}$ & 12.4 \\
\hline Central retinal thickening (disc area) & $N=313-340$ & $\mathrm{~N}=308-336$ & $\mathrm{~N}=307-340$ \\
\hline Baseline, mean (SD) & $8.31(4.15)$ & $8.51(4.21)$ & $7.82(4.28)$ \\
\hline Month 39, mean (SD) & $5.34(4.89)^{* * *} \ddagger$ & $5.38(4.56)^{* * *} \ddagger$ & $6.13(4.59)$ \\
\hline Change from BL to month 39 , mean (SD) & $-2.75(4.46)^{* * *} \ddagger$ & $-2.93(4.03)^{* * *} \ddagger$ & $-1.49(3.74)$ \\
\hline Patients with $\geq 2$-step progression in DRS category from BL (\%) & $\mathrm{N}=324$ & $N=321$ & $\mathrm{~N}=323$ \\
\hline Month 39 & 6.2 & 6.2 & 6.2 \\
\hline Cumulative rate of $\geq 2$-step progression in DRS category from BL (\%) & $\mathrm{N}=324$ & $\mathrm{~N}=321$ & $\mathrm{~N}=323$ \\
\hline Month 39 & $10.2^{*}+\dagger$ & 10.6 & 14.2 \\
\hline \multicolumn{4}{|l|}{ Fluorescein angiography } \\
\hline Macular leakage (disc area) & $N=335-346$ & $N=323-343$ & $N=340-349$ \\
\hline Baseline, mean (SD) & $8.66(4.73)$ & $8.64(4.46)$ & $7.97(4.62)$ \\
\hline Month 39, mean (SD) & $7.68(4.73)$ & $7.42(4.57)$ & $7.57(4.69)$ \\
\hline Change from BL to month 39, mean (SD) & $-0.89(4.10)$ & $-1.06(3.71)$ & $-0.38(3.18)$ \\
\hline Macular capillary loss (disc area) & $\mathrm{N}=300-334$ & $\mathrm{~N}=291-337$ & $\mathrm{~N}=311-340$ \\
\hline Baseline, mean (SD) & $0.24(0.66)$ & $0.19(0.65)$ & $0.16(0.42)$ \\
\hline Month 39, mean (SD) & $0.34(0.83)$ & $0.35(1.06)$ & $0.28(0.93)$ \\
\hline Change from BL to month 39 , mean (SD) & $+0.13(0.52)$ & $+0.17(0.61)$ & $+0.15(0.81)$ \\
\hline \multicolumn{4}{|c|}{$\begin{array}{l}{ }^{*} \mathrm{p}<0.05 \text { vs sham; }{ }^{* *} \mathrm{p}<0.01 \text { vs sham; }{ }^{* * *} \mathrm{p}<0.001 \text { vs sham. } \\
\text { †Based on an analysis of covariance model with treatment and study as fixed effects and baseline value as a covariate. } \\
\text { †Based on an analysis of covariance model with treatment as a fixed effect and baseline value as a covariate. } \\
\text { SCSME is defined as retinal thickening } \geq 1 \text { disc area, part } \leq 1 \text { disc diameter from macula centre or retinal thickening or adjacent hard exudates } \leq 500 \mu \mathrm{m} \text { from macula centre. } \\
\text { IBased on Wilcoxon rank-sum test. } \\
+† \text { Based on the log-rank test. } \\
\text { AUC, area under curve; BL, baseline; CSME, clinically significant macular oedema; DEX, dexamethasone; DRS, diabetic retinopathy severity. }\end{array}$} \\
\hline
\end{tabular}


Figure 2 Mean change from baseline in macular volume versus time. $\mathrm{p} \leq 0.002$ at all time points for dexamethasone intravitreal (DEX) implants versus sham (analysis of covariance with treatment as a fixed effect and baseline value as covariate). $B$, baseline; F, final visit.

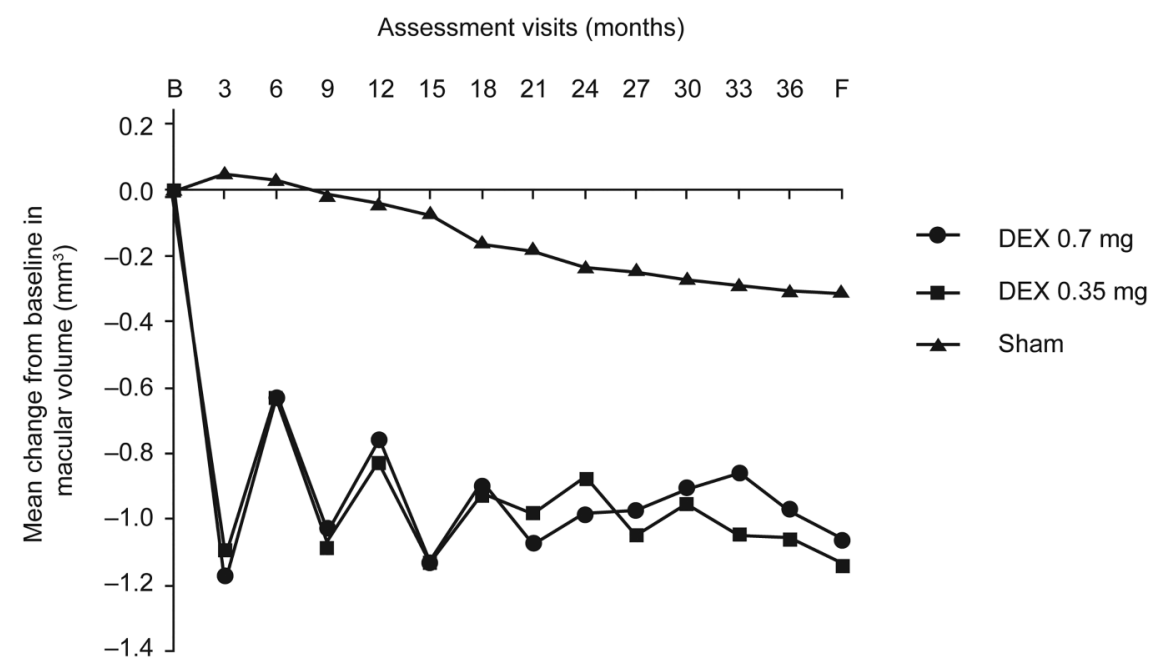

Compared with sham-treated patients, DEX implant-treated patients showed a delay of $\sim 12$ months in onset of two-step progression in diabetic retinopathy severity (10th percentile of time to progression was $\sim 36$ months for both the DEX implant $0.7 \mathrm{mg}$ and $0.35 \mathrm{mg}$ treatment groups vs $\sim 24$ months for the sham treatment group; $p=0.03$ and $p=0.08$, respectively). The 10th percentile of time to two-step improvement in diabetic retinopathy severity was $\sim 24$ and $\sim 13$ months for the DEX implant 0.7 and $0.35 \mathrm{mg}$ treatment groups versus $\sim 24$ months for sham $(p=0.655$ and $p=0.364$, respectively).

The prevalence of CSME declined steadily in both DEX implant treatment groups, and more gradually in the sham group. At study end, fewer DEX implant $0.7-$ and $0.35 \mathrm{mg}$-treated eyes had CSME than sham-treated eyes $(p<0.05$ and $p<0.01$, respectively; table 2). More DEX implant $0.7-$ and $0.35 \mathrm{mg}$-treated eyes showed an improvement in CSME (shift from a higher to a lower grade) between baseline and study end than sham-treated eyes $(\mathrm{p}<0.05$ and $\mathrm{p}<0.01$, respectively; table 2$)$.

\section{Fluorescein angiography findings}

DEX implant $0.7 \mathrm{mg}$ - and DEX implant $0.35 \mathrm{mg}$-treated eyes showed greater reductions (from baseline) in total area of macular fluorescein leakage than sham-treated eyes during the first year (mean -0.35 and -0.52 vs +0.11 disc areas at 6 months, both $\mathrm{p}<0.05$ vs sham; mean -0.58 and -0.79 vs -0.03 disc areas at 12 months, $\mathrm{p}<0.01$ for DEX implant $0.35 \mathrm{mg}$ vs sham). However, these effects were modest and were not sustained; at study end the reductions in total area of fluorescein leakage in the DEX implant treatment groups were not significantly different from those of the sham group (table 2). Changes (from baseline) in total area of macular capillary loss did not differ significantly between DEX implant- and sham-treated eyes at any time (table 2).

\section{DISCUSSION}

This pooled analysis of data from the MEAD trials ${ }^{13}$ is the first to examine the long-term retinal changes associated with the visual acuity improvements produced by intravitreal DEX implant in DME. DEX implant, administered with a median frequency of four to five injections over a 3 -year period, provided sustained improvements in OCT and fundus photography-based anatomical markers of macular oedema, whereas macular fluorescein leakage showed improvement only over the first year. Compared with sham-treated eyes, DEX implant-treated eyes displayed significant decreases in CSRT, macular volume and central retinal thickening at all study time points, with treatment differences emerging as early as month 3 . There was no significant effect on macular capillary loss.

The present findings extend the limited anatomical information provided by earlier short-term phase II studies of DEX implant 0.7 and $0.35 \mathrm{mg}$ in patients with DME. ${ }^{12}{ }^{16}{ }^{17} \mathrm{In}$ keeping with previous studies suggesting that the efficacy of DEX implant on CSRT peaks at approximately 1-3 months before gradually declining, ${ }^{11} 1216$ the profile of mean change in CSRT versus time observed in the present study was characterised by a saw-tooth pattern (figure 1), with each cycle of improvement presumably corresponding to retreatment with DEX implant. A similar saw-tooth pattern was obtained with observed data. This pattern is most likely explained by the gradual decline over time in DEX release from the implant in situ, and suggests that a retreatment interval of less than 6 months may be required for a more consistent anatomical response.

The reduction in macular fluorescein leakage noted during year 1 is consistent with findings from previous short-term, phase II studies. ${ }^{12} 1617$ However, the reason for the discrepancy between the longer-term OCT and fluorescein angiography findings is unclear. Although fluorescein angiography is a reliable method for qualitative assessment of fluid leakage, a reduction in fluorescein intensity is not always accompanied by a reduction in area of fluorescein leakage. Likewise, fluorescein angiography findings do not always mirror OCT findings in DME: in some cases, macular leakage may be evident in the absence of an increase in retinal thickness; in other cases, fluorescein angiography may fail to detect intraretinal or subretinal fluid that is evident on OCT. ${ }^{18}$ Additionally, the diffuse fluorescein staining of non-cystoid oedema in DME may be below the detection threshold of the OCT instrument. ${ }^{18} 19$

Strengths of the present study include its large patient population, its extended duration and inclusion of a sham treatment arm, investigator and patient masking to treatment and a range of end points. The study design ensured that treatment outcomes were not complicated by the effects of adjunctive DME therapies. However, the requirement for subjects requiring adjunctive treatment to exit the study resulted in high attrition, particularly in the control group, which may have adversely affected ITT/LOCF analyses. Other potential study limitations include the lack of adjustment for glycaemic and blood pressure control, which may affect macular thickness, ${ }^{20}$ and the lower reproducibility of macular thickness measurements obtained 
with TD-OCT, the standard technology when the study was initiated (2004), compared with current spectral-domain OCT instruments. ${ }^{21}$ Variability is also likely to have arisen from substitution of centre-point thickness for CSRT owing to scan quality issues and possibly from the natural diurnal variation in macular thickness in eyes with DME. ${ }^{22}$ Nevertheless, manual centre-point thickness measurements performed by the reading centre operators showed excellent reproducibility.

In summary, this pooled data analysis extends the evidence from previous studies in DME, indicating that DEX implant 0.7 and $0.35 \mathrm{mg}$ provides sustained improvements in anatomical measures of macular oedema over a 3-year treatment period. DEX implant thus has the potential to reduce the need for laser therapy and provides an alternative to anti-VEGF therapy in DME.

Acknowledgements Writing and editorial assistance was provided to the authors by Andrew Fitton, PhD, of Evidence Scientific Solutions (Horsham, UK). The full list of participating investigators is published in the primary manuscript.

Contributors RPD and X-YL are guarantors for this paper. All authors participated in the study design, data interpretation and drafting, critical review, revision and approval of the manuscript. HC conducted the data analysis. The study sponsor participated in the design of the study, data analysis and data interpretation and preparation, review and approval of the manuscript.

Funding The study and medical writing support were sponsored by Allergan, Inc., Irvine, California, USA.

Competing interests RPD and SS have received grant support and consulting fees from Allergan, Inc. X-YL and YH are employees of Allergan, Inc. At the time of the study, HC and SMW were employees of Allergan, Inc.

Ethics approval As this was a multicentre study, several IECS and IRBs approved the study.

Provenance and peer review Not commissioned; externally peer reviewed.

Data sharing statement For additional unpublished data from the study, Allergan should be contacted.

Open Access This is an Open Access article distributed in accordance with the Creative Commons Attribution Non Commercial (CC BY-NC 4.0) license, which permits others to distribute, remix, adapt, build upon this work non-commercially, and license their derivative works on different terms, provided the original work is properly cited and the use is non-commercial. See: http://creativecommons.org/ licenses/by-nc/4.0/

\section{REFERENCES}

1 Klaassen I, Van Noorden CJ, Schlingemann RO. Molecular basis of the inner blood-retinal barrier and its breakdown in diabetic macular edema and other pathological conditions. Prog Retin Eye Res 2013;34:19-48.

2 Adamis AP, Berman AJ. Immunological mechanisms in the pathogenesis of diabetic retinopathy. Semin Immunopathol 2008;30:65-84.

3 Bhagat N, Grigorian RA, Tutela A, et al. Diabetic macular edema: pathogenesis and treatment. Surv Ophthalmol 2009;54:1-32.
4 Ehrlich R, Harris A, Ciulla TA, et al. Diabetic macular oedema: physical, physiological and molecular factors contribute to this pathological process. Acta Ophthalmol 2010;88:279-91.

5 Tang J, Kern TS. Inflammation in diabetic retinopathy. Prog Retin Eye Res 2011;30:343-58.

6 Boyer DS, Hopkins JJ, Sorof J, et al. Anti-vascular endothelial growth factor therapy for diabetic macular edema. Ther Adv Endocrinol Metab 2013;4:151-69.

7 Song SJ, Wong TY. Current concepts in diabetic retinopathy. Diabetes Metab J 2014;38:416-25.

8 Bandello F, Cunha-Vaz J, Chong NV, et al. New approaches for the treatment of diabetic macular oedema: recommendations by an expert panel. Eye 2012;26:485-93.

$9 \mathrm{Kim}$ YH, Choi MY, Kim YS, et al. Triamcinolone acetonide protects the rat retina from STZ-induced acute inflammation and early vascular leakage. Life Sci 2007;81:1167-73.

10 Tamura H, Miyamoto K, Kiryu J, et al. Intravitreal injection of corticosteroid attenuates leukostasis and vascular leakage in experimental diabetic retina. Invest Ophthalmol Vis Sci 2005;46:1440-4.

11 Pacella $E$, Vestri AR, Muscella $R$, et al. Preliminary results of an intravitreal dexamethasone implant (Ozurdex $®$ ) in patients with persistent diabetic macular edema. Clin Ophthalmol 2013;7:1423-8.

12 Callanan DG, Gupta S, Boyer DS, et al., for the Ozurdex PLACID Study Group. Dexamethasone intravitreal implant in combination with laser photocoagulation for the treatment of diffuse diabetic macular edema. Ophthalmology 2013;120:1843-51.

13 Boyer DS, Yoon YH, Belfort R Jr, et al., for the Ozurdex MEAD Study Group. Three-year, randomized, sham-controlled trial of dexamethasone intravitreal implant in patients with diabetic macular edema. Ophthalmology 2014;121:1904-14.

14 [No authors listed]. Grading diabetic retinopathy from stereoscopic color fundus photographs - an extension of the modified Airlie House classification. ETDRS report number 10. Early Treatment Diabetic Retinopathy Study Research Group. Ophthalmology 1991;98(Suppl 5):786-806.

15 [No authors listed]. Classification of diabetic retinopathy from fluorescein angiograms. ETDRS report number 11. Early Treatment Diabetic Retinopathy Study Research Group. Ophthalmology 1991;98(Suppl 5):807-22.

16 Boyer DS, Faber D, Gupta S, et al., for the Ozurdex CHAMPLAIN Study Group. Dexamethasone intravitreal implant for treatment of diabetic macular edema in vitrectomized patients. Retina 2011;31:915-23.

17 Haller JA, Kuppermann BD, Blumenkranz MS, et al. Randomized controlled trial of an intravitreous dexamethasone drug delivery system in patients with diabetic macular edema. Arch Ophthalmol 2010;128:289-96.

18 Kozak I, Morrison VL, Clark TM, et al. Discrepancy between fluorescein angiography and optical coherence tomography in detection of macular disease. Retina 2008:28:538-44

19 Otani T, Kishi S. Correlation between optical coherence tomography and fluorescein angiography findings in diabetic macular edema. Ophthalmology 2007;114:104-7.

20 Stratton IM, Kohner EM, Aldington SJ, et al., for the UKPDS Group. UKPDS 50: risk factors for incidence and progression of retinopathy in type II diabetes over 6 years from diagnosis. Diabetologia 2001;44:156-63.

21 Leung CK, Cheung CY, Weinreb RN, et al. Comparison of macular thickness measurements between time domain and spectral domain optical coherence tomography. Invest Ophthalmol Vis Sci 2008;49:4893-7.

22 Frank RN, Schulz L, Abe K, et al. Temporal variation in diabetic macular edema measured by optical coherence tomography. Ophthalmology 2004;111:211-17. 